\title{
Arteriovenous Access Monitoring with Ultrasound Dilution in a Pediatric Hemodialysis Unit
}

\author{
Isa F. Ashoora, blizabeth A. Hughson ${ }^{a}$ Michael J.G. Somers ${ }^{a, b}$ \\ a Boston Children's Hospital and ${ }^{b}$ Harvard Medical School, Boston, Mass., USA
}

\section{Key Words}

Ultrasound dilution - Vascular access · Hemodialysis .

Quality improvement of $A V$ access flow is very sensitive in detecting hemodynamically significant stenosis and can decrease AV access thrombosis rates.

C 2015 S. Karger AG, Basel

\begin{abstract}
Background: Permanent arteriovenous (AV) access is the preferred access for dialysis delivery in children and adolescents requiring chronic hemodialysis (HD). Ultrasound dilution (UD) monitoring of AV access flow is widely used in adult HD units for the early detection of stenosis but experience in pediatrics is limited. Methods: We monitored all maintenance HD patients with AV access using a noninvasive screening algorithm based on UD access flow. We assessed the effectiveness of this algorithm by comparing it to fistulagrams and its impact on AV access-related morbidity. Results: $A V$ access thrombosis rates fell from 13.5 per 100 patient-months on HD during the baseline period to 3.5 per 100 patient-months on HD during the screening period $(p<$ 0.04). The mean blood flow rate by UD measurement was lower in $\mathrm{AV}$ accesses that went on to thrombose compared to those without thrombosis $\left(1,203 \mathrm{ml} / \mathrm{min} / 1.73 \mathrm{~m}^{2} \mathrm{vs} .1,683\right.$ $\left.\mathrm{ml} / \mathrm{min} / 1.73 \mathrm{~m}^{2}, \mathrm{p}<0.001\right)$. When compared to fistulagrams, the screening algorithm was $94 \%$ sensitive and $77 \%$ specific in detecting hemodynamically significant stenosis, with positive and negative predictive values of 83 and $91 \%$ respectively. Conclusions: A noninvasive UD screening algorithm
\end{abstract}

(C) 2015 S. Karger AG, Base

0253-5068/15/0393-0093\$39.50/0

\section{Introduction}

In children and adolescents receiving maintenance hemodialysis (HD), permanent vascular access using an arteriovenous graft (AVG) or arteriovenous fistula (AVF) is preferred over central venous catheters (CVCs) [1]. While AVF and AVG are associated with fewer infectious complications and tend to function longer than CVCs, they do develop neointimal hyperplasia that leads to stenosis and thrombosis $[2,3]$. Such complications interrupt the optimal provision of dialysis and may require CVC placement. Various vascular access surveillance strategies have been described in adults [4], but there is limited experience in pediatric patients. The pediatric vascular access clinical guidelines published by the National Kidney Foundation Kidney Disease Outcome Quality Initiative (KDOQI) recommend establishing a surveillance strategy to detect access stenosis and direct patients for early intervention [1].

For many years, our pediatric HD patients underwent scheduled surveillance fistulagrams. While minimally in-

\section{KARGER 125}

E-Mail karger@karger.com

www.karger.com/bpu
Michael J.G. Somers, MD

Boston Children's Hospital

Division of Nephrology

300 Longwood Avenue, Boston, MA 02115 (USA)

E-Mail michael.somers@ childrens.harvard.edu 
vasive, such procedures were costly, required radiation exposure, and, in younger children, sedation and anesthesia. These issues prompted us to introduce monthly ultrasound dilution (UD) monitoring of AV access to replace surveillance fistulagrams. Initially this change was adopted as part of a quality improvement initiative to reduce access complications. In this report, we discuss the impact of this new screening strategy on detecting AV access stenosis and improving thrombosis rates and also assess the utility of UD as a screening tool in pediatric dialysis patients.

\section{Methods}

\section{Participants and Quality Improvement Plan}

We included all patients receiving maintenance HD using an AVG or AVF from January 2007 through June 2011. Age at ESRD diagnosis, gender, weight, body surface area (BSA), length of time on $\mathrm{HD}, \mathrm{AV}$ access type, thrombosis, hospitalizations related to AV access complications, and need for temporary dialysis catheter placement were recorded. The 24 months from January 2007 through December 2008 served as the baseline period prior to our intervention. AV access surveillance during that period consisted of surveillance fistulagrams every 3 to 6 months and as clinically indicated for possible access dysfunction. The 30 -month period from January 2009 through June 2011 constituted the intervention period when, at least every month, we utilized UD monitoring of AV access blood flow in all patients and as indicated following clinical events or procedures. This routine was modeled after a prior, successful short-term UD monitoring trial in a pediatric dialysis unit [5-7]. A single nurse was designated the vascular access resource specialist and obtained all measurements. Each patient initially had several UD measurements to establish a baseline access blood flow. Measurements were reviewed on a monthly basis by a team comprised of a pediatric nephrologist, a vascular surgeon, an interventional radiologist, and a vascular access nurse. Patients with an absolute AV access blood flow less than $600 \mathrm{ml} /$ min or with a greater than $20 \%$ decrease in access flow relative to their baseline (even if greater than $600 \mathrm{ml} / \mathrm{min}$ ) were referred for a fistulagram.

Our primary objective was to evaluate the impact of UD monitoring on AV access-related morbidity, notably access thrombosis. Secondary morbidity outcome variables included hospitalization related to access complications and requirement for new access creation or temporary dialysis catheter placement.

Our secondary objective was to evaluate the utility of UD monitoring as a screening tool for hemodynamically significant AV stenosis. To accomplish this, we set out to evaluate three questions: (1) Can UD monitoring differentiate between patent AV accesses and those at true risk to thrombose? (2) Can UD monitoring detect an improvement in blood flow following interventional procedures to restore patency? (3) What is the sensitivity and specificity of UD monitoring for detecting hemodynamically significant stenosis compared to fistulagrams?

The Boston Children's Hospital Institutional Review Board approved the study.

\section{Ultrasound Dilution Monitoring Method}

Measurements were obtained during the first $60 \mathrm{~min}$ of dialysis. The vascular access nurse used standardized techniques and protocol recommended by the manufacturer (Transonic Systems, Ithaca, N.Y., USA). Briefly, the device utilizes two reusable sensors calibrated for pediatric blood lines, with a sensor attached to both venous and arterial lines while the patient receives HD. The UD monitor interprets changes in Doppler velocity in relation to the dialyzer blood flow. For each measurement, the blood pump is stopped and the lines are reversed to create recirculation. Dialysis is resumed without ultrafiltration with blood flow set between 250 and $300 \mathrm{ml} / \mathrm{min}$. When a signal from the UD monitor is observed, a saline bolus is introduced into the venous line. Blood flow results are reported in $\mathrm{ml} / \mathrm{min}$.

\section{Contrast Venography (Fistulagrams)}

All patients identified by UD monitoring at risk for AV access dysfunction (criteria listed above) underwent fistulagrams within $48 \mathrm{~h}$. Additional fistulagrams were obtained for clinical indications including abnormal access physical exam, ongoing difficult cannulation or excessive bleeding, and elevated venous pressures. On venography, stenoses involving at least $50 \%$ narrowing were considered hemodynamically significant and those patients underwent angioplasty and possible stenting.

\section{Statistical Analysis}

Demographic variables were compared between the baseline and intervention cohorts using Fisher's exact test, Mann-Whitney test, or Student's $t$ test for categorical and continuous variables as indicated. AV access-related complication rates were calculated for each individual patient based on episode count relative to the length of time on HD. Composite rates from each cohort were calculated and expressed as mean event rate per patient month on hemodialysis and the means of the groups compared for statistically significant differences using Mann-Whitney test.

$\mathrm{AV}$ access flow rates from patients who experienced at least one thrombotic event were averaged and expressed in both raw format $(\mathrm{ml} / \mathrm{min})$ and corrected to BSA $\left(\mathrm{ml} / \mathrm{min} / 1.73 \mathrm{~m}^{2}\right)$ and compared to flow rates from patients with patent AV accesses using MannWhitney test. In patients who experienced a thrombotic event, the mean AV access flow rates, before and after interventional procedures to restore patency, were compared using the paired $t$ test. All statistical analysis was performed using GraphPad software with $\mathrm{p}<0.05$ considered statistically significant.

\section{Results}

\section{Study Participants}

The baseline cohort consisted of 14 patients (5 AVF, 9 AVG) and the intervention cohort 16 patients (7 AVF, 9 AVG). Since their time on dialysis overlapped both periods, 8 patients ( $3 \mathrm{AVF}, 5 \mathrm{AVG}$ ) from the baseline cohort were also members of the intervention cohort. There were no statistically significant differences in age at ESRD diagnosis, gender, weight, BSA, or predominant AV access type between cohorts, even when the overlapping 
Table 1. Cohort characteristics

\begin{tabular}{lcl}
$\begin{array}{l}\text { Baseline cohort }(\mathrm{n}=14) \\
\text { median (range) }\end{array}$ & $\begin{array}{l}\text { Intervention cohort }(\mathrm{n}=16) \\
\text { median (range) }\end{array}$ & $\mathrm{p}$ value \\
\hline 50 & 50 & $>0.9$ \\
$14(4-21)$ & $15.5(4-22)$ & 0.47 \\
$1.62(1.1-2.2)$ & $1.45(1.1-2.2)$ & 0.36 \\
$57(27.5-104.5)$ & $50.6(27.5-101.6)$ & 0.3 \\
$24(2-24)$ & $8(1-29)$ & 0.008 \\
$24(24-24)$ & $11(1-29)$ & 0.02 \\
$14(2-24)$ & $4(2-15)$ & 0.1 \\
\hline
\end{tabular}

Table 2. Summary of UD screening measurements

No. of UD measurements

Access age at 1st UD measurement, median (range) months

UD follow-up duration, median (range) months

UD blood flow in AV access that went on to develop thrombosis, mean (95\% CI) $\mathrm{ml} / \mathrm{min}$

UD blood flow in AV access that remained patent throughout their follow-up, mean $(95 \% \mathrm{CI}) \mathrm{ml} / \mathrm{min}$

UD blood flow in stenosed AV access prior to angioplasty, mean $(95 \% \mathrm{CI}) \mathrm{ml} / \mathrm{min}$

UD blood flow in stenosed AV access post angioplasty, mean $(95 \% \mathrm{CI}) \mathrm{ml} / \mathrm{min}$
162

$12(1-156)$

$5(1-29)$

$903(820-985)$

$1,399(1,289-1,509)$

$730(589-808)$

$1,180(1,026-1,293)$

Table 3. Impact of ultrasound dilution monitoring on AV access-related morbidity

\begin{tabular}{lll}
\hline Primary objective outcome measures & Baseline group $(\mathrm{n}=14)$ & Intervention group (n=16) \\
\hline $\begin{array}{l}\text { Thrombosis events, mean rate* } \\
\text { AV access-related complications (hospitalizations, new } \\
\text { access, temporary HD catheter placement), mean rate* }\end{array}$ & 13.5 per 100 patient-months on HD 3.5 per 100 patient-months on HD $<0.04$ \\
\hline
\end{tabular}

* To account for differences in length of time on HD, rates were determined for individual patients in each group based on episode count relative to months on HD. Each group's patient rates were then averaged and means compared.

patients were censored from analysis. The intervention cohort had a shorter median duration on hemodialysis compared to the baseline cohort ( 8 vs. 24 months, $\mathrm{p}=$ $0.008)$. This was attributed to renal transplantation or transfer to other dialysis units in the intervention period for 6 of the 8 overlapping patients. Table 1 lists the characteristics for each cohort.

\section{Primary Objective: Impact of Ultrasound Dilution \\ Monitoring on AV Access-Related Morbidity}

Table 2 summarizes UD measurements. During the intervention period, 162 UD access flow measurements were obtained on 16 patients. Median AV access age at UD screening initiation was 12 months, with monitoring continuing for a median of 5 months.

Arteriovenous Access Monitoring
Table 3 summarizes AV access thromboses and complications across both time periods. During the baseline period, 10 patients experienced a total of 24 thrombotic events, yielding a mean AV access thrombosis rate of 13.5 events per 100 patient-months on HD. Following the introduction of UD monitoring, 11 thrombotic events occurred in 4 patients, leading to a reduction in the mean $\mathrm{AV}$ access thrombosis rate to 3.5 events per 100 patientmonths on HD $(\mathrm{p}<0.04)$. The mean rate of other AV access-related complications including hospitalizations, new access creation, and temporary dialysis catheter placement was low in the baseline period, measuring 4 events per 100 patient-months on HD and declined further with screening to 2.5 events per 100 patient-months on $\mathrm{HD}$, though this did not reach statistical significance. 
Secondary Objective: The Utility of Ultrasound

Dilution Monitoring as a Screening Tool for

Hemodynamically Significant AV Access Stenosis

The access flow rate from all patients who experienced at least one thrombotic episode averaged $903 \mathrm{ml} / \mathrm{min}$ (95\% CI $820-985 \mathrm{ml} / \mathrm{min}$ ). This flow rate was significantly lower than in patients with accesses that were always patent, whose average access flow measured $1399 \mathrm{ml} / \mathrm{min}$ ( $95 \%$ CI $1,289-1,509 \mathrm{ml} / \mathrm{min}$ ), $\mathrm{p}$ value $<0.001$. This difference was also consistent when access flow rates were corrected for patient body surface area (BSA) (online suppl. fig. 1; for all online suppl. material, see www.karger.com/ doi/10.1159/000368976).

Following angioplasty, UD monitoring also accurately detected improvement in the AV access flow. The median flow rate increased following intervention from 730 $\mathrm{ml} / \mathrm{min}$ (95\% CI $589-808 \mathrm{ml} / \mathrm{min})$ to $1,180 \mathrm{ml} / \mathrm{min}(95 \%$ CI $1,026-1,293 \mathrm{ml} / \mathrm{min}$ ), $\mathrm{p}$ value $<0.001$ (online suppl. fig. 2).

29 fistulagrams were obtained during the intervention period, 18 (62\%) prompted by the screening algorithm with either an absolute UD flow rate of $<600 \mathrm{ml} / \mathrm{min}$ or a greater than $20 \%$ decline in UD flow from baseline. The other 11 fistulagrams were obtained for other clinical indications. Of the 29 studies, 16 detected hemodynamically significant stenoses that required further intervention. Fifteen of 16 studies were prompted by the screening algorithm (UD flow $<600 \mathrm{ml} / \mathrm{min}$ or $>20 \%$ decline from baseline flow), demonstrating a sensitivity of $93.75 \%$ for this screening approach. Of the 11 fistulagrams obtained for clinical indications with otherwise reassuring UD flows, only one demonstrated significant stenosis, yielding a negative predictive value of $90.9 \%$ (online suppl. table 1).

\section{Discussion}

Permanent AV access should be considered a better alternative to CVCs for chronic hemodialysis in children and adolescents. Permanent AV access results in fewer infectious complications, lower hospitalization rates, and better measures of clearance compared to CVCs [2]. The NKF-DOQI recommends the use of a permanent vascular access for pediatric hemodialysis patients [1], and programs such as the International Pediatric Fistula First (IPFF) initiative have been developed to promulgate these recommendations $[8,9]$.

Given the inherent difficulties in creating permanent vascular access in children due to small vessel size and more limited surgical expertise with pediatric vascular surgery [8], maintenance of these accesses once they have been successfully created becomes all the more important. Current data suggests that AV access in children when closely monitored and maintained can manifest similar rates of ongoing functional adequacy as in the adult population [3].

The importance of good long-term vascular access in children and adolescents on HD is two-fold. Although renal transplantation is the preferred treatment for ESRD in pediatric patients, a recent analysis of a large cohort of US children on maintenance HD with permanent AV access revealed that only $22 \%$ undergo renal transplantation within one year of HD initiation and only $42 \%$ by the end of 4-years [2]. Thus, many children face a prolonged period on $\mathrm{HD}$. Moreover, given that pediatric patients with ESRD have a much longer projected lifespan than adults with ESRD, maintaining a functioning access for as long as possible preserves other vessels for future vascular access.

Studies in adults suggested that AV access surveillance with UD monitoring can help identify patients at risk for future access thrombosis $[10,11]$ and several systematic reviews and meta-analyses of access flow surveillance in adults confirmed that UD monitoring reduces the need for access interventions $[12,13]$. The outcome of access surveillance in a pediatric ESRD population with fewer vascular comorbid conditions such as diabetes could be even more advantageous, both in terms of reduction in the need for interventions and in long-term access function.

Following the successful short-term experience of a single center, pediatric HD unit using UD for AV access monitoring [5-7], our dialysis unit introduced a noninvasive screening algorithm for permanent $\mathrm{AV}$ access monitoring. The algorithm was conceived after the review of available literature and consensus agreement between pediatric nephrologists, vascular surgeons, and interventional radiologists at our hospital. One nurse trained in AV access flow measurement using UD method performed all measurements to eliminate inter-operator variability. We used both an absolute flow rate value below which patients were sent for definitive evaluation by interventional radiology as well as any $20 \%$ decline from baseline flow rate for further evaluation [11, 14].

One of the strengths of our study is its longitudinal duration; in fact, this is the longest AV access flow monitoring intervention in a pediatric HD unit reported to date. It is also the first published pediatric report of successful use of AV access flow trends over time as a surveil-
96

Blood Purif 2015;39:93-98

DOI: $10.1159 / 000368976$
Ashoor/Hughson/Somers 
lance strategy. Over the 30-month intervention period, we successfully reduced AV access-related morbidity compared to the preceding 24 months. AV access thrombosis rate fell significantly from 13.5 episodes per 100 patient-months to 3.5 episodes per 100 patient-months, comparable to the rate reported by Goldstein et al of 4.1 episodes per 100 patient-months during their 12-month intervention [6], and underscoring that this intervention can have long-lived benefits. We also demonstrated a trend toward lower access-related hospitalizations and a reduced need for new access creation or temporary catheter placement, as would be expected, if the primary access can be adequately maintained and stays functional longer. Although our study population contained children with both AVFs and AVGs, the proportion of patients with AVF in the intervention group was not significantly higher than the baseline group, suggesting that the benefits observed are likely attributed to the screening program and not a result of the generally lower complication rates noted with AVF compared to AVG [3].

In addition to these direct clinical benefits, these results also help validate UD monitoring as a reliable screening strategy in children. Our data suggests that UD measurements can discriminate between accesses that are more at an imminent risk of thrombosis and those whose patency is likely longer-lived. We also show that UD successfully detects improved AV access flow following interventional procedures to restore patency. Accordingly, UD monitoring could be a valuable strategy in the post angioplasty period, particularly for AVG where the risk of early restenosis requiring further intervention is higher [14].

Finally, we assessed the accuracy of our screening algorithm by comparing it to the 'gold standard' results obtained from fistulagrams and demonstrated that our 'positive' screening criteria afforded both high sensitivity and predictive values for identifying hemodynamically significant stenosis while maintaining reasonable specificity. In fact, given that we had stopped performing protocol fistulagrams, and that most patients with 'negative' screening results did not undergo further fistulagrams because of absence of other clinical indications, the true specificity for these screening criteria is likely even higher.

A potential limitation of this study is that our algorithm used a raw cut-off of $600 \mathrm{ml} / \mathrm{min}$ regardless of BSA to direct patients for further evaluation by interventional radiology. Given that the majority $(14 / 16)$ of our patients had a BSA $<1.73 \mathrm{~m}^{2}$, corrected AV access flows to a standardized BSA would have resulted in higher flow rates.
Therefore, our screening cut-off may have been more sensitive and less specific than if an AV access flow rate normalized to $1.73 \mathrm{~m}^{2} \mathrm{BSA}$ was used. In real practice, however, only 5 of the 18 'positive' screening tests were picked up because of a raw access flow $<600 \mathrm{ml} / \mathrm{min}$, highlighting the value of monitoring $\mathrm{AV}$ access flow over time and intervening when there is a significant decline $(>20 \%)$ from the baseline flow rate even when the flow is well above a predefined absolute value.

A further limitation in our study is its retrospective observational design in reporting the outcomes of our quality improvement intervention. Although this limits the ability to infer causation with certainty, the intervention itself was a prospective measure that was put in place at the beginning of the 30-month observation period, with monthly multidisciplinary meetings to discuss results and review the safety and efficacy of the algorithm given that scheduled fistulagrams were phased out during this period. Moreover, despite the retrospective nature, all imaging reports and dialysis patient information including AV access flow database were stored in our electronic medical record system, allowing for complete data retrieval and analysis.

Finally, our study is limited by the small sample size reflecting the small census of a typical pediatric dialysis unit and, as a result, limits the power to detect significant differences in morbidity between the two periods.

In summary, we demonstrated the successful longterm implementation of a non-invasive AV access surveillance program in a pediatric hemodialysis unit. This surveillance strategy was highly sensitive and it effectively identified children with permanent $\mathrm{AV}$ access at risk of thrombosis. Widespread adoption of similar UDbased surveillance programs in pediatric hemodialysis units will facilitate collaborative prospective trials to determine the impact of these screening programs on longterm AV access survival and minimization of access-related morbidity.

\section{Acknowledgments}

We would like to thank all the members of the vascular access team as well as the dialysis unit nursing staff at Boston Children's Hospital for their diligent dedication to improving the quality of care provided to our patients.

\section{Disclosure Statement}

None disclosed. 


\section{References}

$>1$ National Kidney Foundation: KDOQI clinical practice guidelines and clinical practice recommendations for 2006 updates: hemodialysis adequacy, peritoneal dialysis adequacy and vascular access. Am J Kidney Dis 2006; 48(suppl 1):S1-S322.

-2 Fadrowski JJ, Hwang W, Neu AM, Fivush BA, Furth SL: Patterns of use of vascular catheters for hemodialysis in children in the United States. Am J Kidney Dis 2009;53:91-98.

$>3$ Sheth RD, Brandt ML, Brewer ED, Nuchtern JG, Kale AS, Goldstein SL: Permanent hemodialysis vascular access survival in children and adolescents with end-stage renal disease. Kidney Int 2002;62:1864-1869.

$\checkmark 4$ Kumbar L, Karim J, Besarab A: Surveillance and monitoring of dialysis access. Int J Nephrol 2012;2012:649735.
5 Goldstein SL, Allsteadt A: Ultrasound dilution evaluation of pediatric hemodialysis vascular access. Kidney Int 2001;59:2357-2360.

6 Goldstein SL, Allsteadt A, Smith CM, Currier $\mathrm{H}$ : Proactive monitoring of pediatric hemodialysis vascular access: effects of ultrasound dilution on thrombosis rates. Kidney Int 2002; 62:272-275.

7 Goldstein SL, Smith CM, Currier H: Noninvasive interventions to decrease hospitalization and associated costs for pediatric patients receiving hemodialysis. J Am Soc Nephrol 2003;14:2127-2131.

$>8$ Chand DH, Valentini RP: International pediatric fistula first initiative: a call to action. Am J Kidney Dis 2008;51:1016-1024.

-9 Chand DH, Bednarz D, Eagleton M, Krajewski L: A vascular access team can increase AV fistula creation in pediatric ESRD patients: a single center experience. Semin Dial 2009;22: 679-683.

10 May RE, Himmelfarb J, Yenicesu M, et al: Predictive measures of vascular access thrombosis: a prospective study. Kidney Int 1997;52: 1656-1662.
11 Neyra NR, Ikizler TA, May RE, et al: Change in access blood flow over time predicts vascular access thrombosis. Kidney Int 1998;54: 1714-1719.

12 Tonelli M, James M, Wiebe N, Jindal K, Hemmelgarn B: Ultrasound monitoring to detect access stenosis in hemodialysis patients: a systematic review. Am J Kidney Dis 2008;51: 630-640.

13 Casey ET, Murad MH, Rizvi AZ, et al: Surveillance of arteriovenous hemodialysis access: a systematic review and meta-analysis. J Vasc Surg 2008;48(5 suppl):48S-54S.

14 Schwab SJ, Oliver MJ, Suhocki P, McCann R: Hemodialysis arteriovenous access: detection of stenosis and response to treatment by vascular access blood flow. Kidney Int 2001;59: 358-362. 